\title{
The structural view of bacterial translocation-specific chaperone SecB: implications for function
}

\author{
Jiahai Zhou and Zhaohui Xu* \\ Department of Biological Chemistry, Medical School and \\ Life Sciences Institute, University of Michigan, 210 \\ Washtenaw Avenue, Ann Arbor, MI 48109-2216, USA.
}

\section{Summary}

$\mathrm{SecB}$ is a molecular chaperone that functions in bacterial post-translational protein translocation pathway. It maintains newly synthesized precursor polypeptide chains in a translocation-competent state and guides them to the translocon via its high-affinity binding to the ligand as well as to the membraneembedded ATPase SecA. Recent advances in elucidating the structures of SecB have enabled the examination of protein function in the structural context. Structures of SecB from both Haemophilus influenzae and Escherichia coli support the early two-subsite polypeptide-binding model. In addition, the detailed molecular interaction between SecB and SecA was revealed by a structure of SecB in complex with the C-terminal zinc-containing domain of SecA. These observations explain the dual role of SecB plays in the translocation pathway, as a molecular chaperone and a specific targeting factor. A model of SecB-SecA complex suggests that the binding of SecA to SecB changes the conformation of the polypeptide binding sites in the chaperone, enabling transfer of precursor polypeptides from SecB to SecA. Recent studies also show the presence of a second zinc-independent SecB binding site in SecA and the new interaction might contribute to the function of SecB.

\section{Introduction}

The role of molecular chaperones in protein folding has been well established over the last decade. In general, molecular chaperones have a particular affinity for nonnative conformations that exist in protein folding intermediates. Interaction between folding intermediates and

Accepted 5 August, 2005. *For correspondence. E-mail zhaohui@ umich.edu; Tel. (+1) 734615 2077; Fax (+1) 7347636492.

(c) 2005 The Authors

Journal compilation (C) 2005 Blackwell Publishing Ltd chaperone stabilizes folding intermediates and prevents them from aggregation. Molecular chaperones also play important roles in protein translocation because the majority of proteins are translocated across the membrane as unfolded polypeptides. Therefore, it is important for them to be kept in an unfolded state to remain competent for translocation.

$\mathrm{SecB}$ is a molecular chaperone specialized in the post-translational protein translocation pathway of some proteobacteria. It binds to newly synthesized precursor polypeptides (preproteins) and stabilizes them in an unfolded and non-aggregated state after they exit from the ribosome translation tunnel (Lecker et al., 1989; 1990; Liu et al., 1989; Breukink et al., 1992). It also delivers preproteins to the membrane-embedded translocon via its specific interaction with SecA, an ATPase that provides part of the actual energy for translocation (Hartl et al., 1990; de Cock and Tommassen, 1992; Hoffschulte et al., 1994; Fekkes et al., 1998). The role of SecB in preprotein export was first revealed by Kumamoto and Beckwith (1983; 1985 ) when they found that $\sec B$ mutations resulted in translocation defects for a subset of secretory proteins in Escherichia coli. Extensive work has been carried out on $\mathrm{SecB}$ as its first identification (Weiss et al., 1988; Kumamoto, 1989). Several excellent reviews on the function of SecB have appeared in the past few years (Randall and Hardy, 1995; 2002; Fekkes and Driessen, 1999; Kim and Kendall, 2000; Driessen, 2001; Driessen et al., 2001). In this review, we will focus on advances in our understanding of the molecular mechanism of SecB function based on recent structural analyses (Xu et al., 2000; Dekker et al., 2003; Zhou and Xu, 2003).

\section{SecB structure}

In solution, SecB exists as a tetramer with a molecular mass of $69 \mathrm{KDa}$. The X-ray crystal structures of SecB from both E. coli and Haemophilus influenzae (Xu et al., 2000; Dekker et al., 2003) have shown that the molecule is organized as a dimer of dimers, consistent with data from biochemical studies (Fig. 1) (Muren et al., 1999; Topping et al., 2001). The monomer unit contains a four-stranded antiparallel $\beta$-sheet followed by a pair of antiparallel $\alpha$ - 
A

1 MSEQKQDVAATEEQQPVLQIQRIYVKDVSFEAPNLPHI FQQEWKPKLGFD

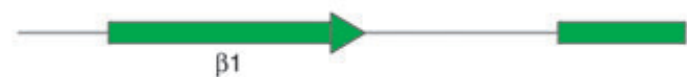

51 LSTETTQVGDDLYEVVLNISVETTLEDSGDVAFICEVKQAGVFTISGLED

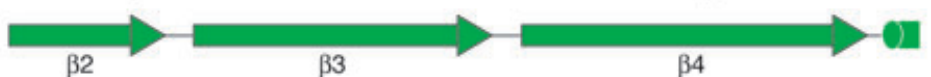

101 VQMAHCLTSQCPNMLFPYARELVSNLVNRGTFPALNLSPVNFDALFVEYM

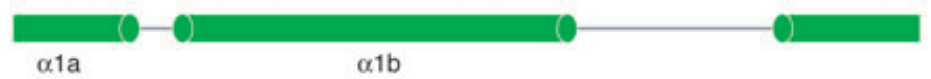

151 NRQQAENAEEKSEEEQTKH 169

$\alpha 2$
Fig. 1. The crystal structure of the SecB molecule.

A. Protein sequence and secondary structure assignment of $H$. influenzae SecB. Secondary structure elements are indicated underneath the sequence: $\alpha$-helices are drawn as cylinders; $\beta$-strands as arrows; and other elements as grey lines.

B. Ribbon drawings of the SecB tetramer are shown in two orthogonal views. Each subunit in the tetramer is coloured differently. The secondary structural elements for the green subunit are labelled as in (A): $\alpha$-helices as coils, $\beta$ strands as arrows and other elements as thick lines. One dimer consists of the green and red subunits while the other consists of the blue and yellow subunits.
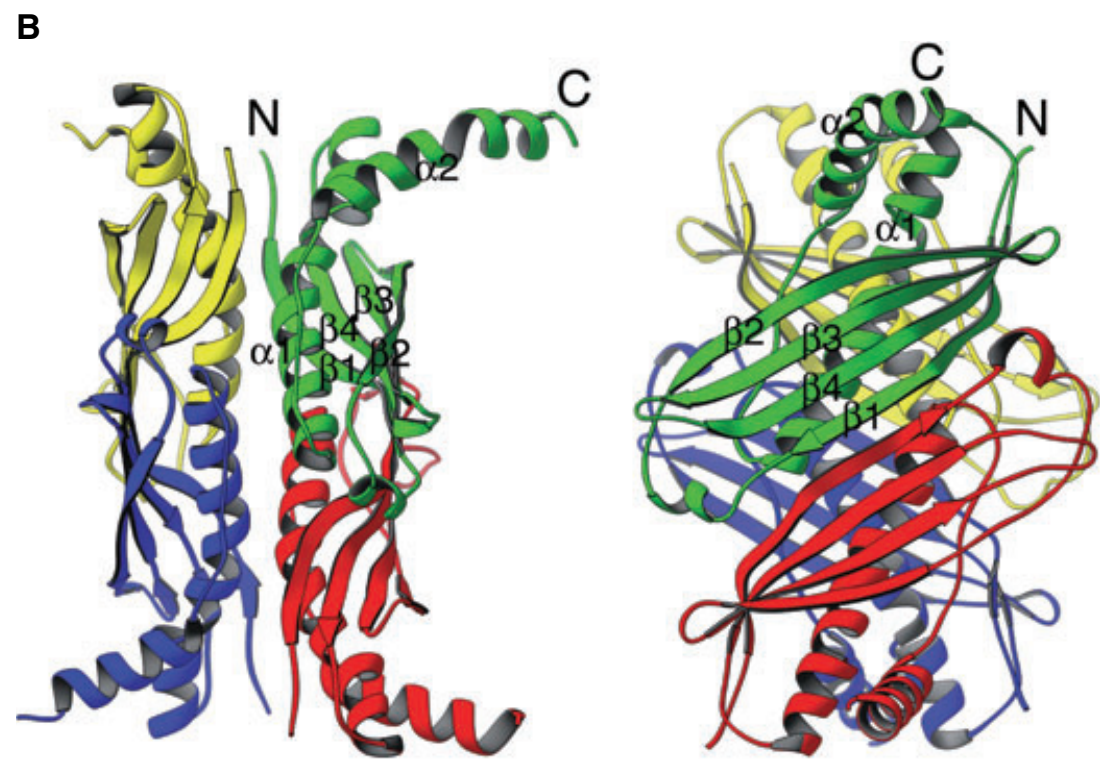

helices. The dimer is a flat molecule formed by a $180^{\circ}$ rotation of one monomer with respect to the other and pairing them together via strand $\beta 1$ and helix $\alpha 1$. As a result of the pairing, there is a surface-exposed antiparallel $\beta$-sheet on one face of the SecB dimer. The dimer is mainly stabilized by main chain hydrogen bonds between the two antiparallel $\beta 1$ strands from the two monomers. Two SecB dimers associate to form a tetramer by sandwiching four long $\alpha 1$ helices between the eight-stranded antiparallel $\beta$-sheets. The tetramer is very stable, with an estimated tetramer-dimer equilibrium constant at $\mathrm{pH} 7.6$ well below $20 \mathrm{nM}$ (Muren et al., 1999). The dimer-dimer interface is stabilized by polar interactions involving sidechains from the four $\alpha 1$ helices.

The amino acid sequence identity between $E$. coli SecB and $H$. influenzae SecB is $59 \%$ and, as expected, the two structures are very similar. Differences between them are found mainly in the loop regions. There is a two-residue difference in the connecting loop between strands $\beta 3$ and
34: residues 76-79 in $H$. influenzae SecB and residues 69-70 in E. coli SecB (Xu et al., 2000; Dekker et al., 2003). This flexible $\beta$-hairpin region is close to the negatively charged SecA-binding surface of SecB (see below) and may affect interaction with SecA.

The extreme C-terminus of SecB subunits is not visible in the electron density maps of either of the crystal structures. Proton nuclear magnetic resonance (NMR) spectroscopy demonstrated that this region is highly mobile (Volkert et al., 1999). Deletion of the C-terminal tail produces a stable truncated $\operatorname{SecB}$ protein that retains its ability to bind to non-native polypeptide in vitro but causes a defect in protein export when overproduced in vivo. The defect can be alleviated by overproduction of SecA, suggesting that the $\mathrm{C}$-terminal tail of SecB may interact with SecA. Deletion of the C-terminal tail also leads to a twofold decrease in affinity for non-native polypeptide (Diamond and Randall, 1997). As peptide binding to SecB protects this tail region from proteolysis (Randall, 1992), 
it is likely that the tail contributes to peptide binding. A structure of SecB-peptide complex will provide the necessary molecular details about how the C-terminal tail of SecB packs against the rest of the structure and regulates the preprotein translocation process.

\section{Polypeptide binding}

In the Sec-dependent pathway, protein translocation cannot occur if newly synthesized polypeptide chains are either folded or aggregated (Randall and Hardy, 1986; Weiss et al., 1988; de Cock et al., 1992). To keep newly synthesized proteins in the translocation-competent state, SecB recognizes the non-native conformation within the mature regions of preproteins and binds with high affinity (the dissociation constant is around $5 \mathrm{nM}$ to $50 \mathrm{nM}$ ) (Randall and Hardy, 1995; Randall et al., 1997; 1998; Topping and Randall, 1997). Although SecB does not directly bind to the leader/signal sequence of preproteins (Randall et al., 1990), the presence of this sequence is nevertheless crucial for export. First, the leader sequence can significantly slow down the rate of spontaneous folding of preproteins and, thereby, increase the probability of binding by SecB (Hardy and Randall, 1991). Second, leader sequences bind to a specific domain of SecA and are thought to be important for the transfer of preproteins from SecB to SecA (Lill et al., 1990; Fekkes et al., 1998; Baud et al., 2002).

Randall and colleagues carried out extensive studies to define the SecB-binding frame within the preprotein. By analysing proteolytic digestion fragments of complexes between SecB and its natural ligands maltose-binding protein, galactose-binding protein or oligopeptide-binding protein, the SecB-binding frame was found to be located in the mature region of the three preproteins and to span a stretch of approximately 150-170 residues (Topping and Randall, 1994; Khisty et al., 1995; Randall and Hardy, 1995; Smith et al., 1997). Based on their early studies of a SecB-binding peptide library (Randall, 1992), a model was proposed for the interaction of $\mathrm{SecB}$ with its ligand (Randall and Hardy, 1995). The model suggests that there are two types of peptide-binding motifs or structures that can be bound by SecB: flexible stretches of polypeptide of approximately 15 residues in length and exposed hydrophobic regions within the non-native polypeptide. The initial interaction occurs at the extended and flexible binding site. Saturation of these binding sites induces a conformational change in SecB that leads to exposure of hydrophobic sites for ligand binding.

Consistent with Randall's hypothesis (Randall and Hardy, 1995), it was proposed that there are two peptide binding subsites in SecB based on the crystal structure of H. influenzae protein (Fig. 2) (Xu et al., 2000). A similar situation was observed more recently in the crystal structure of E. coli SecB (Dekker et al., 2003). Subsite 1 corresponds to the deep section of the peptide-binding channel of SecB and may recognize the hydrophobic and aromatic region within the non-native polypeptide because most of the residues lining subsite 1 are aromatic and conserved. Conformational variation among the different subunits of SecB suggests that this region is structurally flexible and provides a possible explanation for the necessary plasticity for peptide binding. Subsite 1 is large enough to accommodate a hypothesized nine-residue 'SecB-binding motif' (Knoblauch et al., 1999). Subsite 2 is much shallower and more open than subsite 1 . It is there-
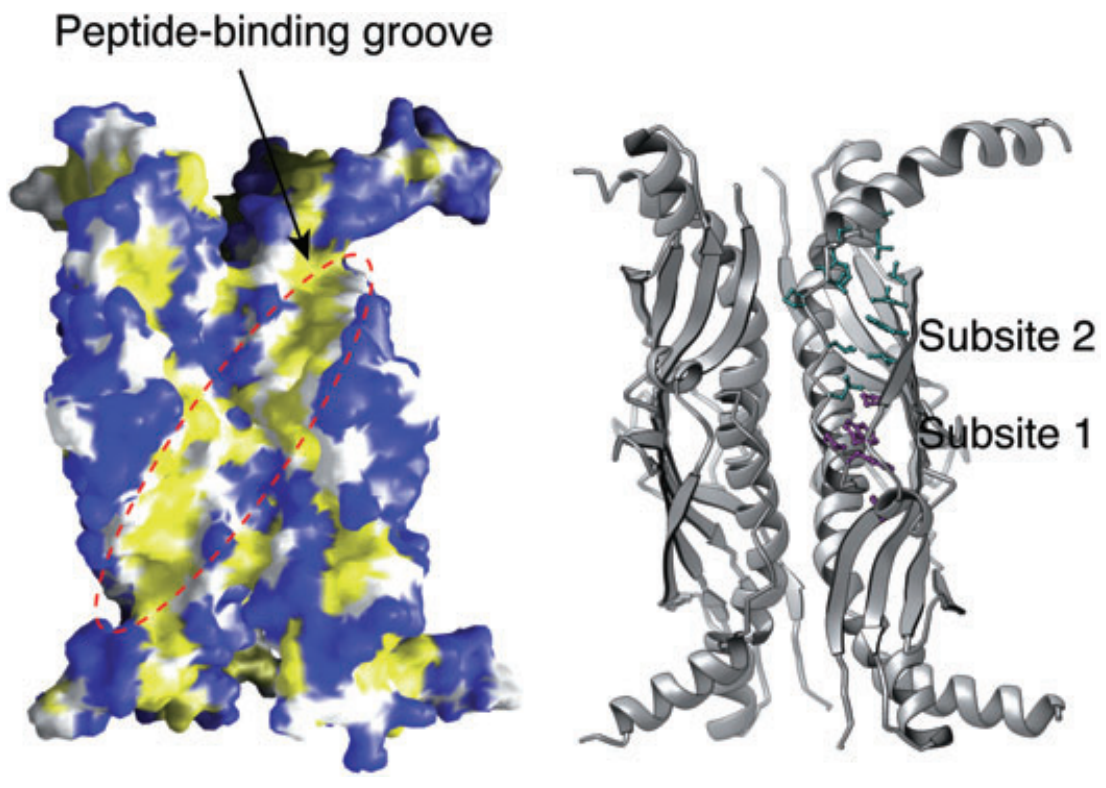

(C) 2005 The Authors

Journal compilation @ 2005 Blackwell Publishing Ltd, Molecular Microbiology, 58, 349-357
Fig. 2. The peptide-binding groove within the SecB molecule. The left panel shows the molecular surface of SecB, coloured according to the underlying atoms: all backbone atoms, white; all non-charged polar and charged sidechain atoms, blue; all hydrophobic side-chain atoms, yellow. The right panel shows the ribbon drawing of SecB in the same orientation as in the left panel. Residues lining the peptidebinding groove in one of the subunits are coloured: subsite 1 , magenta; subsite 2 , cyan. The residues lining the two sites are all hydrophobic. 
fore able to accommodate a more extended region within the non-native polypeptide. In the crystal structure of $H$. influenzae SecB, the $\mathrm{N}$-terminal region of a neighbouring molecule in the crystal lattice inserts itself into subsite 2 (J. Zhou and Z. Xu, unpubl. results). It interacts with subsite 2 by adopting an extended conformation and forming regular main chain hydrogen bonds with strand $\beta 2$ of the $\beta$-sheet. The pattern of interaction likely holds true for the non-native polypeptide ligand.

The molecular symmetry within SecB gives rise to four identical binding sites. While affinity for individual sites might be low, simultaneous occupancy of multiple binding sites will ensure high-affinity binding of polypeptide to SecB. As mentioned above, the SecB-binding frame of its natural ligands spans a stretch of approximately 150-170 residues (Topping and Randall, 1994; Khisty et al., 1995; Smith et al., 1997). While the same SecB molecule does not necessarily occupy the entire stretch of residues, one can speculate that the long unstructured polypeptide segments might wrap around the chaperone to occupy the binding sites on both sides of SecB. It is sufficiently long to loop out from one side through either the path across the top of chaperone or the path across the side of the chaperone. In both cases, the ligand makes close contact with the extreme C-terminal part of SecB. This could explain why polypeptide binding protects these proteasesensitive C-terminal tails (Randall, 1992) and why deletion of the tail part of SecB decreases its affinity for polypeptide ligands (Diamond and Randall, 1997).

\section{SecA recognition}

SecB directs the bound preprotein into the translocation pathway via its specific interaction with membrane-bound SecA (Fekkes et al., 1998). SecB binds to SecA with low affinity in solution (dissociation constant is about $1.6 \mu \mathrm{M}$ ) (den Blaauwen et al., 1997). The binding affinity increases significantly when SecA is bound to the membraneembedded translocon SecYEG complex (the dissociation constant is $10-30 \mathrm{nM}$ ) (Fekkes et al., 1997). The binding is even tighter (dissociation constant around $10 \mathrm{nM}$ ) if SecB is loaded with a polypeptide ligand (Hartl et al., 1990; Fekkes et al., 1997). The SecB binding site on SecA is localized primarily at the extreme C-terminus of SecA, although additional sites have also been suggested (Woodbury et al., 2000) (see below for more discussion). Removal of the C-terminal 22 residues of SecA causes a deficiency in SecB-mediated preprotein translocation (Fekkes et al., 1997).

The crystal structure of $H$. influenzae SecB in complex with the last $27 \mathrm{C}$-terminal residues of $H$. influenzae SecA (SecAc) provides details about the molecular interaction between the two proteins (Fig. 3) (Zhou and Xu, 2003). $\mathrm{SecB}$ uses the solvent-exposed surface of the eightstranded $\beta$-sheet formed by two of the four subunits to interact with one SecAc peptide. In the crystal structure, the SecAc peptide is mainly stabilized by a bound zinc atom. The zinc atom is co-ordinated by three highly conserved cysteines and a histidine. Substitution of these residues in SecA by serine abolishes the ability of SecB to promote preprotein translocation (Rajapandi and Oliver, 1994). The well-structured SecA C-terminal region is necessary for SecB interaction, as the interaction is disrupted by treatment of SecA with a zinc chelator and restored by the addition of $\mathrm{ZnCl}_{2}$ (Fekkes et al., 1999). Two recent solution NMR structures of E. coli SecAc peptide suggest that structural changes in SecAc upon binding to SecB are minimal (Dempsey et al., 2004; Matousek and Alexandrescu, 2004). The crystal structure also shows four residues in $H$. influenzae SecAc, Arg878, Asn879, Lys889 and Lys879, contribute significantly to the binding of SecB. Replacement of any of these residues by alanine in SecA abolishes its binding to SecB (Zhou and $\mathrm{Xu}$, 2003).
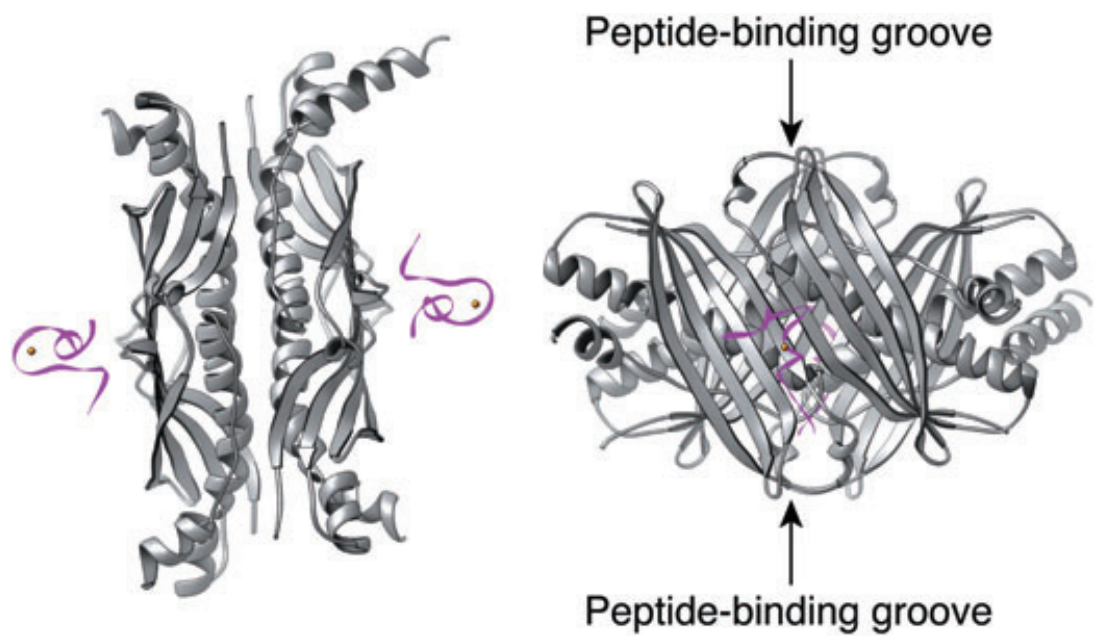

Fig. 3. The crystal structure of the SecBSecAc complex. Ribbon drawings of the complex are shown in two different views. One SecB tetramer (grey) is in complex with two SecA C-terminal peptides (residues 876-899, magenta ribbons). Each SecAc molecule contains one bound zinc ion (golden sphere). 
To visualize how SecB might interact with SecA, the crystal structure of Bacillus subtilis SecA (Hunt et al., 2002) was manually docked onto the structure of H. influenzae SecB-SecAc complex (Fig. 4) (Zhou and $\mathrm{Xu}, 2003)$. Although $B$. subtilis does not possess SecB, high sequence identity (46\%) between SecA molecules from $B$. subtilis and $H$. influenzae suggests that the two proteins are likely to share a very similar overall structure. In addition, a hybrid ' $B$. subtilis SecA' with its C-terminal 27 residues substituted with the corresponding sequence from $H$. influenzae SecA binds to $H$. influenzae SecB (J. Zhou and Z. Xu, unpubl. results). Three major constraints were used in the docking procedure. First, the C-terminal tails of SecA (not seen in any of the three published structures: Hunt et al., 2002; Sharma et al., 2003; Osborne et al., 2004) project into a space beneath the core of the SecA dimer based on the positions of the observed C-terminal ends in the SecA structure. Second, a symmetric complex is assumed to form between SecB and SecA. This requires that the twofold axis of SecA dimer be aligned with the twofold axis relating the two SecA-binding surfaces within a SecB tetramer. Third, the distance between the observed C-terminal ends in the SecA structure and the start of the SecAc segments needs to fit about 15 amino acids missing between these two points of connection. If these 15 amino acids all adopt extended backbone conformations, they could extend over a span of about $50 \AA$. In this model, the distance between the two points of connection (dashed line in
Fig. 4) is $37 \AA$. Although the docking procedure is only approximate, the resulting model allows us to speculate about the interaction between SecB and SecA on a structural term.

The crystal structure of SecA (Fig. 4) (Hunt et al., 2002; Sharma et al., 2003; Osborne et al., 2004) shows that it contains two nucleotide-binding motifs (NBF I and NBF II) with a preprotein cross-linking domain (PPXD) (Kimura et al., 1991) inserted in between at the $\mathrm{N}$ terminal two-thirds of the sequence. The C-terminal onethird of the sequence consists of the $\alpha$-helical scaffold (HSD) and the helical wings domains (HWD) as well as the extreme C-terminal zinc-containing domain. As shown in Figs 4 and 5, SecB-preprotein complex binds to SecA from the bottom direction via the zinc-binding domains of SecA (step 2 in Fig.5). One of the SecB polypeptide-binding grooves located on the top of the molecule is sandwiched between SecB and SecA. This positions SecB-bound preprotein directly beneath the SecA molecule and near its preprotein cross-linking domain, which makes direct preprotein transfer feasible. One could imagine that the simultaneous binding of the C-terminal tails of the SecA dimer to SecB changes the relative orientation of the two eight-stranded $\beta$-sheets in $\mathrm{SecB}$, and therefore the conformation of the polypeptidebinding groove. This could lead to a decrease in the affinity of SecB for the bound preprotein, resulting in the release of preprotein from $\mathrm{Sec} B$ and subsequent transfer to SecA (step 3 in Fig. 5).
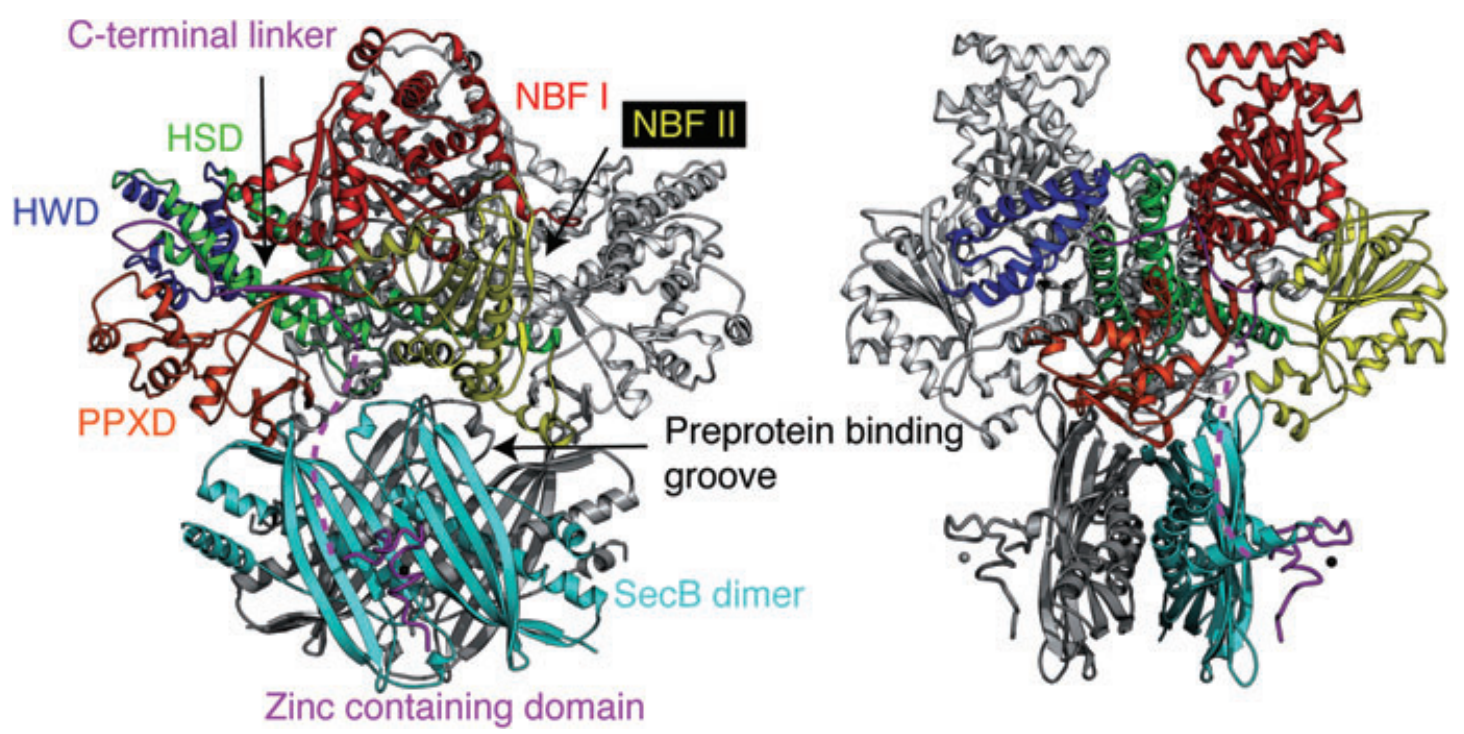

Fig. 4. A hypothetical model of SecA-SecB molecular complex. The hypothetical SecA-SecB complex is shown in two orthogonal views. It was generated by manually docking the C-terminal linker of $B$. subtilis SecA structure onto the N-terminus of $H$. influenzae SecAc of the SecB-SecAc complex structure (see text for details). In both views, SecA dimer is on the top and SecB tetramer is on the bottom. One SecA monomer is coloured white while the other is coloured based on its previously defined domains (Hunt et al., 2002): NBF I, red; PPXD, orange; NBF II, yellow; HSD, green; HWD, blue; and C-terminal linker, magenta. One SecB dimer is coloured grey while the other is coloured cyan and its associated SecAc magenta. Roughly, a total of 15 residues are missing between the visible end of the SecA structure and the beginning of the SecAc structure (dashed line). One of the preprotein-binding grooves of SecB is sandwiched between SecB and SecA. 


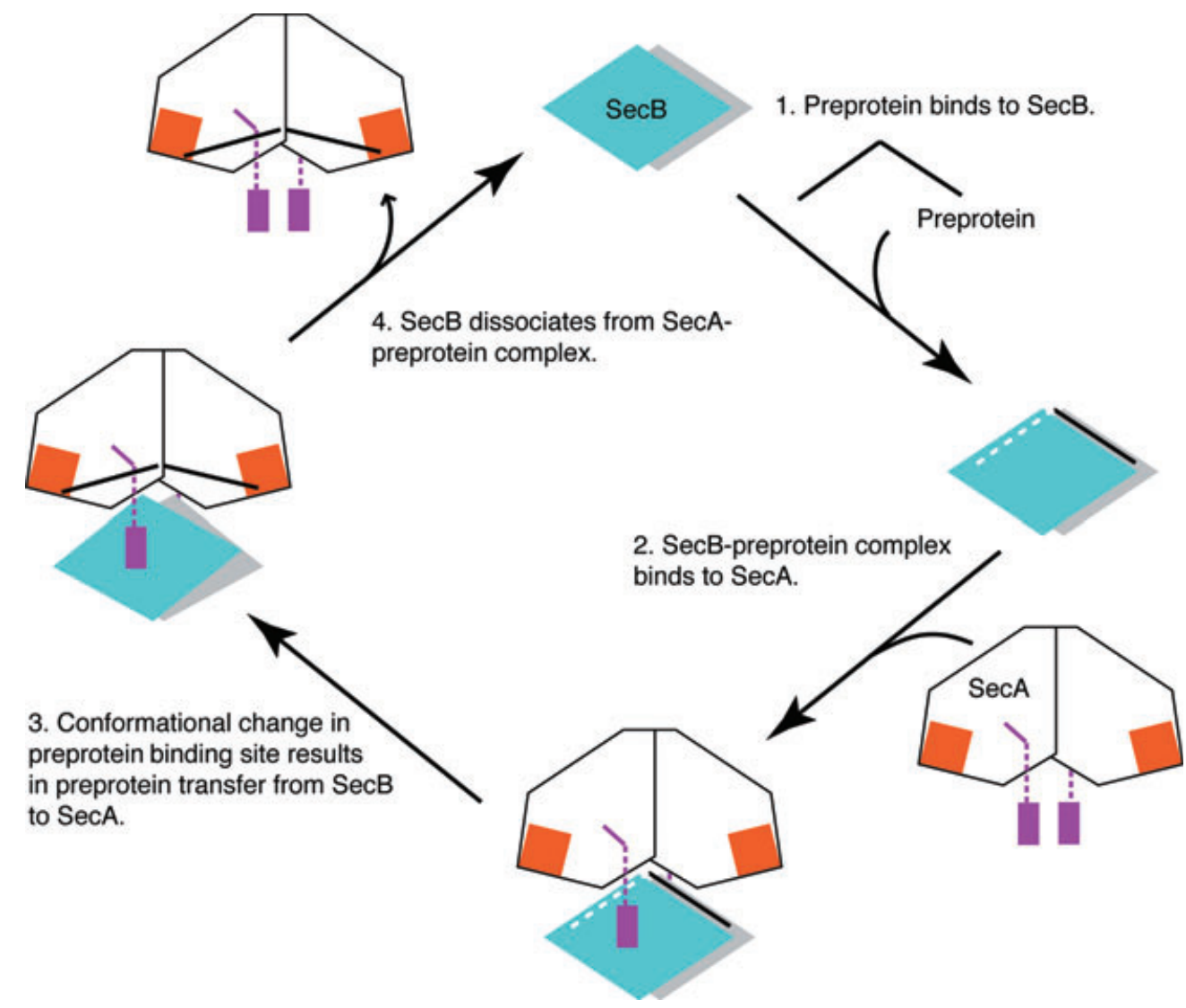

Fig. 5. Model of the SecB-mediated preprotein targeting in the bacterial protein translocation pathway. SecB tetramer is shown as two diamonds coloured cyan and grey. SecA dimer is coloured white except the preprotein cross-linking domains (orange) and the C-terminal zinc-containing domains (magenta). Majority of SecA molecules that interact with SecB-preprotein complex are membrane-associated. It is likely that SecA exists in monomer-dimer equilibrium.

The proposed SecA-SecB interaction model assumes that SecA symmetrically interacts with SecB as a dimer and the interaction is mediated via the $\mathrm{C}$-terminal zinccontaining domain. This is supported by the SecAc-SecB complex structure as well as binding studies using various SecA and SecB mutants (Fekkes et al., 1997; 1999; Zhou and $\mathrm{Xu}, 2003$ ). In particular, both C-terminal tails of SecA dimer appear to be necessary to form a tight SecA-SecB complex as heterodimers of wild-type SecA and a Cterminal truncated form of SecA are defective in SecB binding (Fekkes et al., 1997). Recent studies using chimeric SecA proteins also suggest that the $\mathrm{C}$-terminal tail of $\operatorname{Sec} A$ is necessary and sufficient to mediate the specific interaction between SecB and SecA. B. subtilis lacks a SecB proteins and its SecA does not bind to either $E$. coli or $H$. influenzae SecB. By genetically switching the Cterminal tail of $B$. subtilis SecA to the corresponding part from either $E$. coli SecA or $H$. influenzae SecA, the new hybrid ' $B$. subtilis SecA' can now bind to the cognate $\mathrm{Sec} B$, depending on which tail the chimeric protein contains (J. Zhou and Z. Xu, unpubl. data).
In addition to the above-mentioned interaction, Randall and colleagues discovered a second site of SecA-SecB interaction. By analysing complexes formed between $E$. coli SecA and variants of $E$. coli SecB, they found that the translocation defective variants of SecB still form a complex with SecA, even though they cannot interact with the C-terminal tail of SecA (Woodbury et al., 2000). This hypothesis was further supported by their titration calorimetry and sedimentation velocity centrifugation data (Randall et al., 2004). The second binding site was now mapped to the C-terminal $\alpha$-helix of SecB and the dimer interfacial region of SecA (Randall et al., 2005). They also showed that this additional interaction between SecA and SecB might serve to destabilize the SecA dimer, leading to the asymmetric interaction between the two proteins. Most of the asymmetric binding observed by Randall and colleagues requires the disruption of the interaction involving the zinc-binding domain. It is unclear to what extent this secondary binding contributes to the overall interaction between two wild-type proteins. However, at a certain stage of the SecB function cycle, SecA-SecB interaction 
must be weakened to ensure the release of SecB from the membrane. This would facilitate the $\mathrm{C}$-terminal helix of SecB to interact with the dimer interface of SecA. It is possible that the interaction then weakens the SecA dimer and leads to conformational changes in SecA, which might also contribute to the transfer of preprotein from SecB to SecA (Jilaveanu et al., 2005; Or et al., 2005; Randall et al., 2005). To fully address the functional role of the C-terminal independent SecA-SecB interaction will require the determination of its structure.

\section{Other functions of SecB}

Besides its function in the Sec-dependent protein translocation pathway, SecB is also the chaperone for the secretion of the HasA haemophore through the type I protein translocation pathway in Serratia marcescens (Delepelaire and Wandersman, 1998; Sapriel et al., 2003). HasA is a protein secreted by the Gram-negative bacteria under iron starvation conditions to assist the utilization of external haem as an iron source. Efficient secretion of HasA requires the protein in an unfolded state, which is maintained by the binding of SecB. In vitro studies have shown that SecB specifically binds to unfolded HasA and slows down its folding rate significantly (Wolff et al., 2003). Therefore, it is likely that $\mathrm{SecB}$ uses the hydrophobic polypeptide-binding groove to recognize unfolded HasA. Elimination of SecB in S. marcescens affects the secretion of both HasA and proteins translocated through the Secdependent pathway (Sapriel et al., 2003). Although the chaperoning mechanism of SecB in the two pathways appears to be similar, the downstream interaction partners are different. The SecB residues implicated in SecA binding are not important for HasA secretion. Further biochemical and structural characterization of the type I pathway-specific protein-protein interactions in SecB will help elucidate the mechanism.

SecB may also act as a general chaperone, as it can bind to denatured luciferase and facilitates the subsequent refolding of the protein by the DnaK/DnaJ system (Knoblauch et al., 1999). Recent studies showed that overproduction of SecB can suppress the temperaturesensitive and the aggregation-prone phenotypes caused by elimination of both DnaK and trigger factor in E. coli (Ullers et al. 2004). It should be noted that this reflects a rather aberrant condition in which the cognate folding machinery is highly compromised, while a rescue is observed only upon overproduction of SecB. Whether this reflects a genuine in vivo function of SecB remains to be seen. The structural basis for this activity of $\mathrm{SecB}$ is unknown but likely involves the polypeptide binding site used in protein translocation pathway.

SecB has also been shown to interact directly with bacterial ribosome-bound chaperone trigger factor $(\mathrm{Ha}$ et al., 2004). The dissociation constant between E. coli SecB and $E$. coli trigger factor is $\sim 6 \mu \mathrm{M}$, as determined by surface plasmon resonance. However, complex formation between the two proteins was not observed in a gel filtration chromatography experiment using purified materials. Isothermal titration calorimetry suggested that the dissociation constant is much lower (in the mM range) (J. Zhou and Z. Xu, unpubl. results). If they do interact, which part of SecB structure is involved in interaction? Little is known for the physiological role this interaction might play in vivo. Could it be possible that SecB receives the newly synthesized preprotein from trigger factor rather than through random collision in the cytosol? Clearly, further studies are necessary to clarify these issues.

\section{Conclusions}

The recent crystal structures of SecB and its complex with the C-terminal tail of SecA have shed new light on the molecular mechanism by which the small bacterial chaperone SecB functions in protein translocation. SecB employs hydrophobic, solvent-exposed surfaces to stabilize the non-native conformation that exists in preproteins. The predominant interactions between SecB and preproteins involve non-specific main chain hydrogen bonds and hydrophobic interactions. As SecB does not seem to recognize any particular sequence motifs within preproteins, this structural feature of SecB ensures that different preprotein ligands can be recognized. In contrast, the interaction between SecB and SecA is highly specific. Not surprisingly, the protein-protein interface within the SecBSecA complex comprises highly conserved residues. Interactions between the two proteins are mediated by specific side-chain hydrogen bonds. Replacement of these residues by alanines has a drastic effect on the stability of the molecular complex.

As neither crystal structure contains polypeptide ligands, the exact mode of SecB-polypeptide interaction is not known. In particular, the structural basis for the function of SecB C-terminus in protein translocation is not clear. However, it is not difficult to imagine that a large stretch of polypeptide binds to the hydrophobic SecB peptide-binding groove in an extended conformation. The structure of SecB-SecAc complex suggests that SecA likely modulates the conformation of the polypeptide-binding groove upon its interaction with $\mathrm{SecB}$, thereby promoting the release of preproteins from SecB. While the Cterminal tail of SecA plays a critical role in determining the specific interaction with $\mathrm{SecB}$, other structural elements have also been shown to promote complex formation independent of the C-terminal tail. Elucidating the structure of SecB in complex with full-length SecA is the next logical step to further our understanding of this important event in bacterial protein translocation. 


\section{Acknowledgements}

This work was supported by an R01 grant from US National Institutes of Health Grant (GM60997). Z.X. is a University of Michigan Biological Sciences Scholar and a Pew Scholar in Biomedical Sciences.

\section{References}

Baud, C., Karamanou, S., Sianidis, G., Vrontou, E., Politou, A.S., and Economou, A. (2002) Allosteric communication between signal peptides and the SecA protein DEAD motor ATPase domain. J Biol Chem 277: 13724-13731.

den Blaauwen, T., Terpetschnig, E., Lakowicz, J.R., and Driessen, A.J. (1997) Interaction of SecB with soluble SecA. FEBS Lett 416: 35-38.

Breukink, E., Kusters, R., and De Kruijff, B. (1992) In-vitro studies on the folding characteristics of the Escherichia coli precursor protein prePhoE. Evidence that SecB prevents the precursor from aggregating by forming a functional complex. Eur J Biochem 208: 419-425.

de Cock, H., and Tommassen, J. (1992) SecB-binding does not maintain the translocation-competent state of prePhoE. Mol Microbiol 6: 599-604.

de Cock, H., Overeem, W., and Tommassen, J. (1992) Biogenesis of outer membrane protein PhoE of Escherichia coli. Evidence for multiple SecB-binding sites in the mature portion of the PhoE protein. J Mol Biol 224: 369-379.

Dekker, C., de Kruijff, B., and Gros, P. (2003) Crystal structure of SecB from Escherichia coli. J Struct Biol 144: 313319.

Delepelaire, P., and Wandersman, C. (1998) The SecB chaperone is involved in the secretion of the Serratia marcescens HasA protein through an $\mathrm{ABC}$ transporter. EMBO $\mathrm{J}$ 17: 936-944.

Dempsey, B.R., Wrona, M., Moulin, J.M., Gloor, G.B., Jalilehvand, F., Lajoie, G., et al. (2004) Solution NMR structure and $\mathrm{X}$-ray absorption analysis of the $\mathrm{C}$-terminal zincbinding domain of the SecA ATPase. Biochemistry 43: 9361-9371.

Diamond, D.L., and Randall, L.L. (1997) Kinetic partitioning. Poising SecB to favor association with a rapidly folding ligand. J Biol Chem 272: 28994-28998.

Driessen, A.J. (2001) SecB, a molecular chaperone with two faces. Trends Microbiol 9: 193-196.

Driessen, A.J., Manting, E.H., and van der Does, C. (2001) The structural basis of protein targeting and translocation in bacteria. Nat Struct Biol 8: 492-498.

Fekkes, P., and Driessen, A.J. (1999) Protein targeting to the bacterial cytoplasmic membrane. Microbiol Mol Biol Rev 63: 161-173.

Fekkes, P., van der Does, C., and Driessen, A.J. (1997) The molecular chaperone SecB is released from the carboxyterminus of SecA during initiation of precursor protein translocation. EMBO J 16: 6105-6113.

Fekkes, P., de Wit, J.G., van der Wolk, J.P., Kimsey, H.H., Kumamoto, C.A., and Driessen, A.J. (1998) Preprotein transfer to the Escherichia coli translocase requires the cooperative binding of $\mathrm{SecB}$ and the signal sequence to SecA. Mol Microbiol 29: 1179-1190.

Fekkes, P., de Wit, J.G., Boorsma, A., Friesen, R.H., and
Driessen, A.J. (1999) Zinc stabilizes the SecB binding site of SecA. Biochemistry 38: 5111-5116.

Ha, S.C., Lee, T.H., Cha, S.S., and Kim, K.K. (2004) Functional identification of the SecB homologue in Methanococcus jannaschii and direct interaction of SecB with trigger factor. Biochem Biophys Res Commun 315: 1039-1044.

Hardy, S.J., and Randall, L.L. (1991) A kinetic partitioning model of selective binding of nonnative proteins by the bacterial chaperone SecB. Science 251: 439-443.

Hartl, F.U., Lecker, S., Schiebel, E., Hendrick, J.P., and Wickner, W. (1990) The binding cascade of SecB to SecA to SecY/E mediates preprotein targeting to the E. coli plasma membrane. Cell 63: 269-279.

Hoffschulte, H.K., Drees, B., and Muller, M. (1994) Identification of a soluble SecA/SecB complex by means of a subfractionated cell-free export system. J Biol Chem 269: 12833-12839.

Hunt, J.F., Weinkauf, S., Henry, L., Fak, J.J., McNicholas, P., Oliver, D.B., and Deisenhofer, J. (2002) Nucleotide control of interdomain interactions in the conformational reaction cycle of SecA. Science 297: 2018-2026.

Jilaveanu, L.B., Zito, C.R., and Oliver, D. (2005) Dimeric SecA is essential for protein translocation. Proc Natl Acad Sci USA 102: 7511-7516.

Khisty, V.J., Munske, G.R., and Randall, L.L. (1995) Mapping of the binding frame for the chaperone SecB within a natural ligand, galactose-binding protein. J Biol Chem 270: 25920-25927.

Kim, J., and Kendall, D.A. (2000) Sec-dependent protein export and the involvement of the molecular chaperone SecB. Cell Stress Chaperones 5: 267-275.

Kimura, E., Akita, M., Matsuyama, S., and Mizushima, S. (1991) Determination of a region in SecA that interacts with presecretory proteins in Escherichia coli. J Biol Chem 266: 6600-6606.

Knoblauch, N.T., Rudiger, S., Schonfeld, H.J., Driessen, A.J., Schneider-Mergener, J., and Bukau, B. (1999) Substrate specificity of the SecB chaperone. J Biol Chem 274: 34219-34225.

Kumamoto, C.A. (1989) Escherichia coli SecB protein associates with exported protein precursors in vivo. Proc Natl Acad Sci USA 86: 5320-5324.

Kumamoto, C.A., and Beckwith, J. (1983) Mutations in a new gene, secB, cause defective protein localization in Escherichia coli. J Bacteriol 154: 253-260.

Kumamoto, C.A., and Beckwith, J. (1985) Evidence for specificity at an early step in protein export in Escherichia coli. $J$ Bacteriol 163: 267-274.

Lecker, S., Lill, R., Ziegelhoffer, T., Georgopoulos, C., Bassford, P.J., Jr, Kumamoto, C.A., and Wickner, W. (1989) Three pure chaperone proteins of Escherichia coli - SecB, trigger factor and GroEL - form soluble complexes with precursor proteins in vitro. EMBO J 8: 2703-2709.

Lecker, S.H., Driessen, A.J., and Wickner, W. (1990) ProOmpA contains secondary and tertiary structure prior to translocation and is shielded from aggregation by association with SecB protein. EMBO J 9: 2309-2314.

Lill, R., Dowhan, W., and Wickner, W. (1990) The ATPase activity of SecA is regulated by acidic phospholipids, SecY, and the leader and mature domains of precursor proteins. Cell 60: 271-280. 
Liu, G., Topping, T.B., and Randall, L.L. (1989) Physiological role during export for the retardation of folding by the leader peptide of maltose-binding protein. Proc Natl Acad Sci USA 86: 9213-9217.

Matousek, W.M., and Alexandrescu, A.T. (2004) NMR structure of the C-terminal domain of SecA in the free state. Biochim Biophys Acta 1702: 163-171.

Muren, E.M., Suciu, D., Topping, T.B., Kumamoto, C.A., and Randall, L.L. (1999) Mutational alterations in the homotetrameric chaperone $\mathrm{SecB}$ that implicate the structure as dimer of dimers. J Biol Chem 274: 19397-19402.

Or, E., Boyd, D., Gon, S., Beckwith, J., and Rapoport, T. (2005) The bacterial ATPase SecA functions as a monomer in protein translocation. J Biol Chem 280: 90979105.

Osborne, A.R., Clemons, W.M., Jr, and Rapoport, T.A. (2004) A large conformational change of the translocation ATPase SecA. Proc Natl Acad Sci USA 101: 1093710942.

Rajapandi, T., and Oliver, D. (1994) Carboxy-terminal region of Escherichia coli SecA ATPase is important to promote its protein translocation activity in vivo. Biochem Biophys Res Commun 200: 1477-1483.

Randall, L.L. (1992) Peptide binding by chaperone SecB: implications for recognition of nonnative structure. Science 257: 241-245.

Randall, L.L., and Hardy, S.J. (1986) Correlation of competence for export with lack of tertiary structure of the mature species: a study in vivo of maltose-binding protein in E. coli. Cell 46: 921-928.

Randall, L.L., and Hardy, S.J. (1995) High selectivity with low specificity: how SecB has solved the paradox of chaperone binding. Trends Biochem Sci 20: 65-69.

Randall, L.L., and Hardy, S.J. (2002) SecB, one small chaperone in the complex milieu of the cell. Cell Mol Life Sci 59: 1617-1623.

Randall, L.L., Topping, T.B., and Hardy, S.J. (1990) No specific recognition of leader peptide by $\mathrm{SecB}$, a chaperone involved in protein export. Science 248: 860-863.

Randall, L.L., Topping, T.B., Hardy, S.J., Pavlov, M.Y., Freistroffer, D.V., and Ehrenberg, M. (1997) Binding of SecB to ribosome-bound polypeptides has the same characteristics as binding to full-length, denatured proteins. Proc Natl Acad Sci USA 94: 802-807.

Randall, L.L., Hardy, S.J., Topping, T.B., Smith, V.F., Bruce, J.E., and Smith, R.D. (1998) The interaction between the chaperone SecB and its ligands: evidence for multiple subsites for binding. Protein Sci 7: 2384-2390.

Randall, L.L., Crane, J.M., Liu, G., and Hardy, S.J. (2004) Sites of interaction between SecA and the chaperone SecB, two proteins involved in export. Protein Sci 13: 1124-1133.

Randall, L.L., Crane, J.M., Lilly, A.A., Liu, G., Mao, C., Patel, C.N., and Hardy, S.J. (2005) Asymmetric binding between
SecA and SecB two symmetric proteins: implications for function in export. J Mol Biol 348: 479-489.

Sapriel, G., Wandersman, C., and Delepelaire, P. (2003) The SecB chaperone is bifunctional in Serratia marcescens: $\mathrm{SecB}$ is involved in the Sec pathway and required for Has $A$ secretion by the ABC transporter. J Bacteriol 185: 80-88.

Sharma, V., Arockiasamy, A., Ronning, D.R., Savva, C.G., Holzenburg, A., Braunstein, M., et al. (2003) Crystal structure of Mycobacterium tuberculosis SecA, a preprotein translocating ATPase. Proc Natl Acad Sci USA 100: 22432248.

Smith, V.F., Hardy, S.J., and Randall, L.L. (1997) Determination of the binding frame of the chaperone SecB within the physiological ligand oligopeptide-binding protein. Protein Sci 6: 1746-1755.

Topping, T.B., and Randall, L.L. (1994) Determination of the binding frame within a physiological ligand for the chaperone SecB. Protein Sci 3: 730-736.

Topping, T.B., and Randall, L.L. (1997) Chaperone SecB from Escherichia coli mediates kinetic partitioning via a dynamic equilibrium with its ligands. J Biol Chem 272: 19314-19318.

Topping, T.B., Woodbury, R.L., Diamond, D.L., Hardy, S.J., and Randall, L.L. (2001) Direct demonstration that homotetrameric chaperone SecB undergoes a dynamic dimer-tetramer equilibrium. J Biol Chem 276: 7437-7441.

Ullers, R.S., Luirink, J., Harms, N., Schwager, F., Georgopoulos, C., and Genevaux, P. (2004) SecB is a bona fide generalized chaperone in Escherichia coli. Proc Natl Acad Sci USA 101: 7583-7588.

Volkert, T.L., Baleja, J.D., and Kumamoto, C.A. (1999) A highly mobile C-terminal tail of the Escherichia coli protein export chaperone SecB. Biochem Biophys Res Commun 264: 949-954.

Weiss, J.B., Ray, P.H., and Bassford, P.J., Jr (1988) Purified secB protein of Escherichia coli retards folding and promotes membrane translocation of the maltose-binding protein in vitro. Proc Natl Acad Sci USA 85: 8978-8982.

Wolff, N., Sapriel, G., Bodenreider, C., Chaffotte, A., and Delepelaire, P. (2003) Antifolding activity of the SecB chaperone is essential for secretion of HasA, a quickly folding ABC pathway substrate. J Biol Chem 278: 38247-38253.

Woodbury, R.L., Topping, T.B., Diamond, D.L., Suciu, D., Kumamoto, C.A., Hardy, S.J., and Randall, L.L. (2000) Complexes between protein export chaperone SecB and SecA. Evidence for separate sites on SecA providing binding energy and regulatory interactions. J Biol Chem 275: 24191-24198.

Xu, Z., Knafels, J.D., and Yoshino, K. (2000) Crystal structure of the bacterial protein export chaperone secB. Nat Struct Biol 7: 1172-1177.

Zhou, J., and Xu, Z. (2003) Structural determinants of SecB recognition by SecA in bacterial protein translocation. Nat Struct Biol 10: 942-947. 